\title{
Enseñanza y aprendizaje desde el E-learning. Implicaciones desde la percepción de estudiantes y profesores
}

\author{
Teaching and learning from E-learning. Implications from the \\ perception of students and teachers
}

- OTTO MAGDALENO GONZÁLEZ

ottoalexandergonzalez@gmail.com

Código ORCID: 0000-0001-7410-2147

Universidad Pedagógica Experimental Libertador, UPEL, Venezuela

Artículo recibido en abril 2020 / Arbitrado en mayo 2020 / Publicado en julio 2020

Resumen El propósito fue analizar las implicaciones de las percepciones de los docentes y estudiantes universitarios de Educación Musical de la Universidad Pedagógica Experimental Libertador sobre los procesos de enseñanza y de aprendizaje, basados en E-learning. La metodología se enfocó en el paradigma cualitativo y diseño de campo. La técnica fue la entrevista, la cual se aplicó a docentes y estudiantes de los cursos a distancia. Como resultado se obtuvo que la mayoría de los participantes señalaron que emplean las herramientas de las TIC en los procesos educativos a distancia, tales como: Skype, WhatsApp, Facebook, Twitter, Instagram, mensajes de texto, e-mail, entre otras, además de que estas herramientas proporcionan la posibilidad de interrelacionarse para desarrollar un aprendizaje colaborativo. Con el E-learning se fortalecen y potencian los procesos de enseñanza y aprendizaje porque son mayores las posibilidades para acceder a las distintas modalidades (presencial, a distancia y mixta).

Palabras clave: E-Learning; enseñanza; aprendizaje; herramientas de las TIC; nivel universitario; Educación Musical

\begin{abstract}
The purpose was to analyze the implications of the perceptions of teachers and university students of Musical Education of the Universidad Pedagógica Experimental Libertador on teaching and learning processes, based on e-learning. The methodology focused on qualitative paradigm, supported by a field design. The technique was the interview, which was applied to teachers and students of distance courses. As a result, it was obtained that most of the participants indicated that they use ICT tools in distance education processes, such as: Skype, WhatsApp, Facebook, Twitter, Instagram, text messages, e-mail, etc.; In addition to these tools provide the possibility to interrelate to develop collaborative learning. With E-learning, the teaching and learning processes are strengthened and enhanced because the possibilities to access the different modalities (face-to-face, distance and mixed) are greater.
\end{abstract}

Keywords: $\quad$ E-Learning; teaching; learning; ICT tools; university level; Musical Education 


\section{INTRODUCCIÓN}

Ante los continuos cambios en el mundo es necesario que las personas asuman los retos que aparecen diariamente en todos los aspectos de la vida así como en el ámbito de la educación que es la rama del saber del ser pensante no escapa de esta realidad.

En el nivel universitario, la educación es, generalmente, presencial pues los estudiantes y el docente deben trasladarse desde un punto geográfico hasta la institución educativa. Para algunos la movilización es fácil, pero para otros no por múltiples factores de la dinámica de vida que incide sobre el ellos. En consecuencia, se busca aprovechar otra forma de recibir la enseñanza para que el estudiante pueda sobrellevar ese día a día y así cumplir con su formación. De allí que hayan surgido otras modalidades de estudio y es así como emerge la educación a distancia, que facilita la distribución de tiempo, evita desplazarse hacia el centro educativo y sobre todo facilita que la persona pueda organizarse y construir su propio aprendizaje.

En educación no presencial existen diversas herramientas tecnológicas o recursos web que se pueden aprovechar con la ayuda de las plataformas tecnológicas y es posible facilitar el autoaprendizaje, uno de ellos es el $E$ learning que permite facilitar los procesos de enseñanza y de aprendizaje, a través de los medios electrónicos.

De allí que el objetivo del presente estudio fue analizar las implicaciones de las percepciones de los docentes y estudiantes de la especialidad de Educación Musical del Instituto Pedagógico de Miranda "José Manuel Siso Martínez" de la Universidad Pedagógica Experimental Libertador
(UPEL), con respecto a la enseñanza y el aprendizaje desde el E-learning.

\section{Educación a distancia}

Para Marcelo (2002), la educación a distancia es considerada como aquella en donde el alumno y el profesor están separados bien sea geográfica o temporalmente o ambos y que utilizan ciertos métodos para comunicarse y aprender. La educación a distancia no es reciente; en realidad ha sido un modo de enseñar y aprender de millares de personas durante más de un siglo. No siempre se aprendió a distancia con el apoyo de los actuales medios electrónicos, sino que esta forma de enseñar y aprender ha evolucionado, en el último siglo, a lo largo de tres grandes generaciones de innovación tecnológica que Garrison (1985) identifica como correspondencia, telecomunicación y telemática.

El sistema de educación a distancia basado en el empleo de la tecnología, ha surgido del acuerdo entre disciplinas educativas y de comunicación que favorecen el pensamiento crítico y reflexivo, propiciando el análisis y la investigación, lo cual es necesario en todas las instituciones educativas de educación superior, en vista de la expansión del conocimiento, lo que favorece la formación democrática y el aprendizaje colaborativo.

La Universidad Pedagógica Experimental Libertador emplea los sistemas de educación a distancia para el desarrollo de los procesos de enseñanza y aprendizaje. En este sentido, se ha designado la Comisión Nacional de Virtualización Académica (COMVA), en el año 2007, cuyo propósito es propiciar el uso de las herramientas tecnológicas actuales en los procesos de enseñanza y aprendizaje en la UPEL. 
En este mismo orden de ideas, el Reglamento del Personal Académico de la UPEL (UPEL, 2016) destaca, en su artículo 2, la importancia del uso de las tecnologías en la educación a distancia en la formación profesional, y las ventajas en cuanto a la flexibilidad que brinda al estudiante. En consecuencia, según el artículo 3, la UPEL tiene la responsabilidad de incorporar la educación a distancia en todos sus institutos y emplear las tecnologías de información y comunicación, a fin de dar mayor oportunidad de ingreso $y$ permanencia a los estudiantes y hacer una educación más inclusiva.

\section{Tecnología de Información y Comunicación (TIC)}

Las tecnologías de Información y Comunicación (TIC) son el conjunto de sistemas y productos que se materializan físicamente por medio de dispositivos informáticos y de interconexión que funcionan internamente por medio de programas que las personas utilizan para llevar a cabo procesos de tratamiento de información y de comunicación. Estos avances tecnológicos varían en atención a los escenarios en los que las personas pueden compartir conocimientos, tal como lo presentan las diversas teorías de aprendizaje (Castro, Casado y Guzmán, 2007; Guzmán, 2005, 2008, 2013).

Las TIC son medios y recursos digitales que permiten recopilar, almacenar, transmitir, procesar y recuperar información electrónicamente, así como el control de aparatos de uso cotidiano en la educación y en las fábricas automatizadas (Castro, Casado y Guzmán, 2007; Guzmán, 2005). Sin embargo, los cambios que se han producido en educación, han hecho que se desarrollen interrogantes en base a cómo se utilizan en sus diferentes modalidades y estilos de enseñanza y aprendizaje (De Marco, 2015). Esto en vista de que las TIC generan un cambio en lo concerniente al lugar, tiempo y nuevas áreas del conocimiento, que vienen a sustituir al currículo tradicional y brindan al estudiante la posibilidad de acceder a la enseñanza sin limitaciones de espacio y tiempo. Esta tendencia ha permitido la aparición de nuevas redes de asociación que son ya tendencias fundamentales que debe asumir la educación superior en Venezuela. Para de este modo superar las limitaciones que tiene la universidad en Venezuela que a opinión de Albornoz (1999) es costosa, pero de baja productividad y pertinencia social.

Contreras (2008) resalta el uso de estos insumos aprovechables alojados en la web, para realizar tareas diferentes tales como: enviar mensajes electrónicos con agregados de materiales digitalizados, imágenes, textos, videos o sonidos y su aplicación de listas de distribución, así como la utilización de salas de chats, foros, videoconferencias, y un sinfín de aplicaciones que proporcionan la enseñanza.

Con base a lo anterior y haciendo una revisión de la opinión de Iniciarte (2007), en lo que se refiere al rol que debe representar el pedagogo como regente de la formación a distancia, este no solo se basa en la enseñanza, sino en constituir la exploración de investigaciones y organizarlas, de tal manera que el profesor como intermediario de las técnicas de la enseñanza se halla en un evento nuevo de la realidad, en una tendencia formidable del hecho educativo, en lo que se hace imprescindible su capacidad en afrontar gestiones tales como: diseñar extractos didácticos apropiados, cooperación de varias disciplinas con ambos 
actores educativos, las TIC como conducción de la información y el apoyo en el espacio-tiempo en la instrucción que redunde en pro del entorno laboral.

Al respecto, al hacer una revisión de autores como Pizarro y Cordero (2013), se tiene que las TIC ayudan a los educadores al aplicar procesos transformadores con el fin de incentivar el interés del educando y fortalecer su instrucción a través del manejo de herramientas tecnológicas tales como: imágenes, sonidos y textos, así como otros elementos pedagógicos que fortalezcan a los diferentes espacios del aprendizaje de los educandos siendo estos: salones en la red, diálogo en presentación digital, buscadores de material de investigación vigente y como otros recursos de interés.

Sobre el uso de las TIC por parte de los estudiantes en sus cursos a distancia, Fernández, Server y Carballo (2006) resaltan que:

El alumno se transforma en participante activo y constructor de su propio aprendizaje y el profesor asume el rol de guía y facilitador de este proceso lo cual varía su forma de interactuar con sus alumnos, la forma de planificar y de diseñar el ambiente de aprendizaje. (p. 1)

Por su parte, Hernández (2010) puntualiza que:

el docente que posee una sólida formación en un campo disciplinar, que aconseja o sugiere y guía la forma en que el estudiante puede abordar determinado contenido; resuelve dudas académicas $y$ evalúa los aprendizajes, fomenta el estudio independiente. (p. 444)
De allí que las nuevas tendencias de la educación deban utilizar las TIC como una herramienta de utilidad para favorecer los procesos de enseñanza y de aprendizaje de los ciudadanos que requiere esta nueva era, en la cual se deben preparar para el intercambio social, cultural y educativo que le permita desenvolverse con mayor facilidad en y para la vida, todo esto enmarcado en la educación a distancia, específicamente el $e$ learning.

Las TIC están cada vez más al alcance de todas las personas, y las escuelas no escapan de ellas, puesto que la tecnología presente en la escuela es una herramienta que posibilita el desarrollo de habilidades en el estudiante, lo que se refleja en un aprendizaje constructivo (Guzmán, 2013). De esta manera, es el estudiante quien se encarga de buscar la información y realiza un análisis crítico de la misma (Papert, citado en Darías, 2001). En esta transformación, no solo están involucrados los estudiantes, sino la sociedad en general quien ahora tiene acceso a usar la tecnología para estar informada, aprender y hasta divertirse, por lo que la educación no puede dejar de lado a la tecnología.

Para describir la influencia que tienen las TIC en educación, se considera la opinión de Kustcher y St.Pierre (2001, p. 31):

- Computadoras y periféricos con contenido digital, dentro de los que se destacan CDROM, calculadora, cámara digital, impresora a color, scanner.

- Información digital: en ellos se encuentran los programas de aplicación y los que administran información, como lo son las páginas Web, base de datos, hoja de cálculo, 
programa de aplicación de procesamiento de palabras.

- Comunicación digital: mensajerías de texto, charla en línea, foros electrónicos, telecopiador, teleconferencia, audio y video.

Agrega Ruíz Bolívar (1999), los siguientes recursos:

Transmisiones vía satélite, sistemas multimedios, hipermedios (tutoriales, hipertexto, sistemas de simulación), comunicación mediante computadoras (bancos de información en línea, grupo o listas electrónicas, sistemas de cartelera electrónica, revistas electrónicas, sistemas de navegación, comunicación audiovisual mediante computadora); se dispone además, de una realidad virtual y sistemas de autoría electrónica entre otros. (p. 3)

Estas posibilidades de comunicación e información, las tienen todas las personas actualmente, y las usan para estar informados, comprar, vender en fin hacer todas sus tareas diarias, en consecuencia, resulta casi inconcebible que alguien en la actualidad no tenga un teléfono o un correo electrónico. Al respecto, los ambientes e-learning surgen como una forma de arquitectura cognitiva, que implican trayectorias de información, puesto que determinan la manera como la información es transmitida a través de un grupo (Hollan, Hutchins y Kirsch, 2000).

\section{E-Learning}

E-learning como sucesor directo de la educación a distancia, según Bermejo (2005); Williams, Nicholas y Gunter (2005); Zenger y Walker (2000), surge a mediados del siglo XIX, y se generaliza en los años 60, destacando que en esa época los materiales impresos para la enseñanza eran difundidos por el correo postal tradicional. González-Videgaray (2007) enuncia que el E-learning no es solo pieza de la educación a distancia, sino que perfectamente se puede integrar con la formación presencial.

Otros investigadores teorizan que la " $\mathrm{e}$ " de E-learning es el uso exclusivo de herramientas electrónicas, sin el uso del internet, así como otros que el E-learning debe estar apoyado en la www (World, Wide, Web). Los dominios o sitios Web, son bases primordiales para desarrollar los procesos de enseñanza y aprendizaje no presenciales. Estas al combinarse con conexión: sincrónica, asincrónica y mixta, se denomina como el e-formación o E-learning.

E-learning consiste en educar y capacitar a través del Internet, es un sistema de formación interactivo para desarrollar programas de enseñanza que hace uso masivo de los medios electrónicos para llegar de manera remota al estudiante. Este sistema ha transformado la educación, abriendo puertas al aprendizaje individual $y$ organizacional en escuelas $y$ empresas ya que es un tipo de enseñanza online que permite la interacción del usuario con el material mediante la utilización de diversas herramientas informáticas.

García-Peñalvo y García (2001) señalan que E-learning no es un sustituto de la educación presencial tradicional, sino que actúa como un elemento que se amolda al usuario dependiendo de sus necesidades y beneficio de su aprendizaje.

En lo que se refiere al campo de la educación a distancia y el uso del E-learning en la actualidad, es normal referirse a la existencia de universidades online y las abiertas; aulas virtuales, sistemas a distancias, entre otras. Además, se 
ofrecen una gama de cursos y carreras en diferentes sistemas de E-learning. Pues bien, la enseñanza con el uso del E-learning permitirá romper las barreras de espacio y tiempo, y transformándose podría transformarse en una opción que consentirá al individuo egresar de una carrera universitaria o instituto, sea esta a nivel nacional $O$ internacional. El mismo estudiante lleva su ritmo de avance, pues la holgura de ese tiempo y espacio, le permitirá desenvolverse mejor salvando los obstáculos de la vida diaria: geográficos, económicos, laboral, familiar, entre otros.

Duart y Sangrá (2000) presentan tres modelos representativos de E-learning en la educación superior, que se han implementado en la universidad con resultados diferentes y significativos a través del tiempo y cada uno con ventajas y desventajas:

- Modelos centrados en los medios y recursos, aparecen como consecuencia de la coyuntura actual del desarrollo tecnológico. El modelo se centra en la herramienta tecnológica que se utiliza, que puede ser una sola o la conjunción de diferentes herramientas y también en el papel poco importante que tendrán los dos máximos protagonistas del acto formativo: el profesor (proveedor de contenidos) y el estudiante (usuario que emplea estos contenidos sin límites de tiempo y espacio).

- Modelos centrados en el profesorado, algunas instituciones de enseñanza superior buscan reproducir los esquemas organizativos presenciales con la ayuda de las TIC. Esto genera modelos metodológicos clásicos, centrados más en la enseñanza que en el aprendizaje, en los que el profesor continúa siendo el único referente válido de transmisión del conocimiento, y centrándose básicamente en las TIC para ampliar el alcance de sus clases. No hay innovaciones, se hace a distancia y a través de dispositivos lo mismo que en una clase presencial.

- Modelos centrados en el estudiante, la mayoría de las instituciones que se dedican a la formación apoyan este tipo de modelo. Sin embargo, esto responde a una tendencia mundial donde se valora positivamente que la institución deje de centrarse en la figura del profesor y se centre en la del estudiante. Estos son los que se basan, fundamentalmente, en el autoaprendizaje o la autoformación.

El enfoque o el uso que se haga de cada uno de estos tres elementos básicos permitirán ubicar cualquier institución en un punto concreto de confluencia. Cuanto más cerca se sitúe de alguno de los tres puntos, más centrado estará el modelo analizado en aquella variable. El autor de esta investigación coincide con Duart y Sangrá (2000) y considera que es obvio decir, que el punto central del esquema tridimensional es el que puede dar la medida de un modelo metodológico equilibrado, aunque no tiene que ser necesariamente el que busque cada institución.

En esta innovadora manera de formación está presente una amalgama de metodologías de instrucción; por consiguiente debido a la no presencia física de ambos actores en el escenario educativo, las etapas interactiva y pre-activa de la instrucción son reemplazadas por un lenguaje de edición, y/o medios mecánicos o electrónicos. 


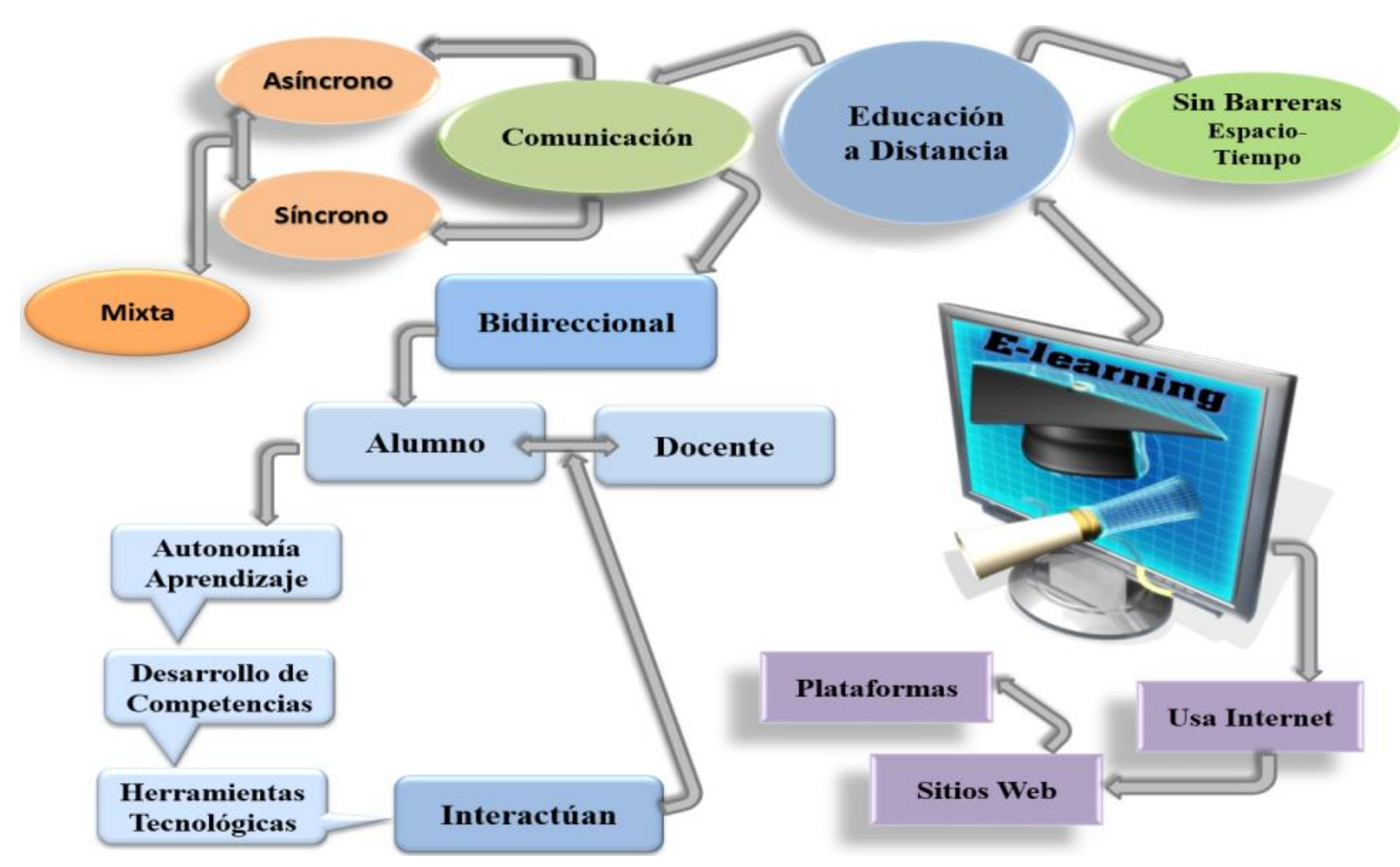

Figura 1. Esquema del E-learning. (Fuente: Elaborado por el autor)

MÉTODO

Investigación desarrollada bajo el enfoque cualitativo emergente, flexible y proyectado. Martínez (2007) plantea que el enfoque cualitativo da razón plena de los comportamientos y manifestaciones de los individuos para identificar la naturaleza profunda de las realidades y de su estructura dinámica. Cada sujeto conoce e interpreta la realidad de manera distinta, sin embargo se pueden presentar evidencias de elementos coincidenciales, convergentes con respecto a otros sujetos. Por otra parte, Cook y Reichardt (1997), señalan que este enfoque promueve estudiar los fenómenos sociales a través de la realidad estudiada en la que se busca un acercamiento más global y comprensivo.

Por otra parte, como estrategia interpretativa, se emplea la hermenéutica, que consiste en una estrategia que permite la interpretación y el discernimiento de algunos escenarios. Sobre todo aquellos relacionados con el ser humano y las relaciones con su entorno, bien sea social, cultural, de forma de vida y la manera de interrelacionarse; y también como sujeto capaz de desarrollar sus potencialidades humanas (Yegres, 2000).

Desde el punto de vista del paradigma cualitativo, la hermenéutica es por lo general trabajada como parte de la metodología de la investigación. En el caso que ocupa el presente trabajo, el enfoque hermenéutico se manifiesta en la interpretación, análisis y comprensión de la información suministrada por los sujetos de investigación y las referencias consultadas. Además, está apoyado en una la investigación de campo la que permitió realizar el diagnóstico, pues se recolectaron los datos 
directamente de la realidad donde ocurren los hechos (Sabino, 1999). En este caso es en el Instituto Pedagógico de Miranda José Manuel Siso Martínez.

Por otra parte, este trabajo responde a las características de una investigación de carácter descriptivo e interpretativo, debido a que se registra la información ordenadamente del hecho investigado. Tamayo y Tamayo (1987) señala que este tipo de investigación "...comprende la descripción, registro, análisis e interpretación de la naturaleza actual, composición o procesos de los fenómenos" (p. 35)

La investigación fue dirigida a los profesores de diferentes especialidades de educación y a los estudiantes de Educación Musical del Instituto Pedagógico de Miranda José Manuel Siso Martínez de la Universidad Pedagógica Experimental Libertador.

La técnica de investigación fue la encuesta, la cual para Villafranca (como se citó en Pérez, 2009 , p. 73) "Consiste en obtener información, opiniones, sugerencias y recomendaciones, mediante técnicas como: la entrevista y el cuestionario". En este mismo orden de ideas Arias (2012) plantea que la encuesta "se define como una técnica que pretende obtener información que suministra un grupo o muestra de sujetos acerca de si mismos, o en relación con un tema en particular" (p. 72).

La encuesta fue realizada a profesores y estudiantes de la Universidad Pedagógica Experimental Libertador, Universidad "Siso Martínez", con el fin de obtener información referente al uso del E-learning como modalidad de enseñanza y aprendizaje para los cursos que se imparten a distancia.

La investigación se organizó en tres fases: a) recolección de la información, b) diagnóstico y recolección de datos y c) análisis de los resultados.

\section{RESULTADOS Y DISCUSIÓN}

Los resultados que emanaron de la investigación permitieron establecer tres categorías (ver Tabla 1), las cuales se analizan a continuación:

Tabla 1. Categorías provenientes de la triangulación de fuentes

\section{Categoría}

E-learning

Herramientas tecnológicas (TIC) usadas en el E-learning

Cursos a distancia ventajas y desventajas

\section{E-learning}

Con respecto a la categoría E-learning para conocer su definición se pudo evidenciar el conocimiento sobre este tópico que tienen determinados grupos de profesores de la UPEL Pedagógico de Miranda "José Manuel Siso
Martínez", pues sus respuestas en relación a lo que entendían como E-learning fueron:

"D1 Lo definiría como una forma de educar, formar y educarse a través de plataformas en la web", 
"D2 Gran ventana al mundo, con grandes posibilidades, que rompe las barreras del tiempo y espacio y convierte de manera virtual el aula",

"D5 Diversas alternativas para propiciar el aprendizaje por medio del uso de herramientas tecnológicas, propiciadas por el docente, con el apoyo y participación activa de sus estudiantes de manera virtual",

"D10 La educación y capacitación a través de Internet",

"D11 Es una forma de educación a través de Internet, es decir utilizando las TICS, herramientas tecnológicas con las cuales no sea imprescindible la presencialidad",

"D12 Es un método que se emplea para administrar cursos bajo la modalidad a distancia con el uso de las TIC, lo cual genera una estrategia de enseñanza apoyada en recursos de la Web".

Cabe señalar que la población docente del "Siso Martínez " a la cual se le aplicó la encuesta de manera aleatoria, algunos profesores declinaron en prestar la colaboración en participar, e indicaron, de manera clara, que a pesar de dictar cursos en la modalidad a distancia no utilizaban ningún tipo de herramientas tecnológicas, como tampoco, la utilización de aulas virtuales.

Por su parte los estudiantes manifestaron no conocer sobre ese término y por consiguiente no emanaron respuesta.

\section{Herramientas tecnológicas usadas en el E-learning}

La categoría herramientas tecnológicas usadas en el E-learning permitió observar que una parte de los pedagogos de la UPEL, Instituto Pedagógico de Miranda "José Manuel Siso Martínez", utilizan una gama de herramientas de las TIC en los procesos educativos a distancia; dentro de los cuales hicieron mención a los chats, foro, blog, MOODLE (la que ofrece la universidad), Google Drive, blogger, Facebook, Movie Macker, (Cubase "programa de secuencia musical" y Finale "Edición y Transcriptor Musical" los profesores de música en su gran mayoría lo operan y las TIC en general), Dropbox, Skype, Youtube, correo electrónico y foros virtuales, aunque por otra parte, hay quienes manifiestan no tener conocimiento, ni del manejo de las herramientas de almacenamiento comunicación y proveedores de información.

Se evidencia, que no todo el personal docente de la universidad utiliza herramientas tecnológicas en sus clases no presenciales, solo algunos de ellos manejan una cantidad importante de estos recursos tecnológicos. Queda entonces la incertidumbre que el personal académico del "Siso Martínez" tengan tal debilidad en el uso de estas tecnologías, pues esta es la punta del iceberg en los cursos que se imparten a distancia por todos los beneficios que estas representan en el aprendizaje de los educandos, como también en la distribución del tiempo a utilizar y de la no presencialidad en la institución que es el clamor de los educandos de la especialidad de música por sus múltiples actividades musicales que realizan.

Se detallan a continuación las herramientas que manejan y las que usan los profesores de la universidad (ver figura 2): 


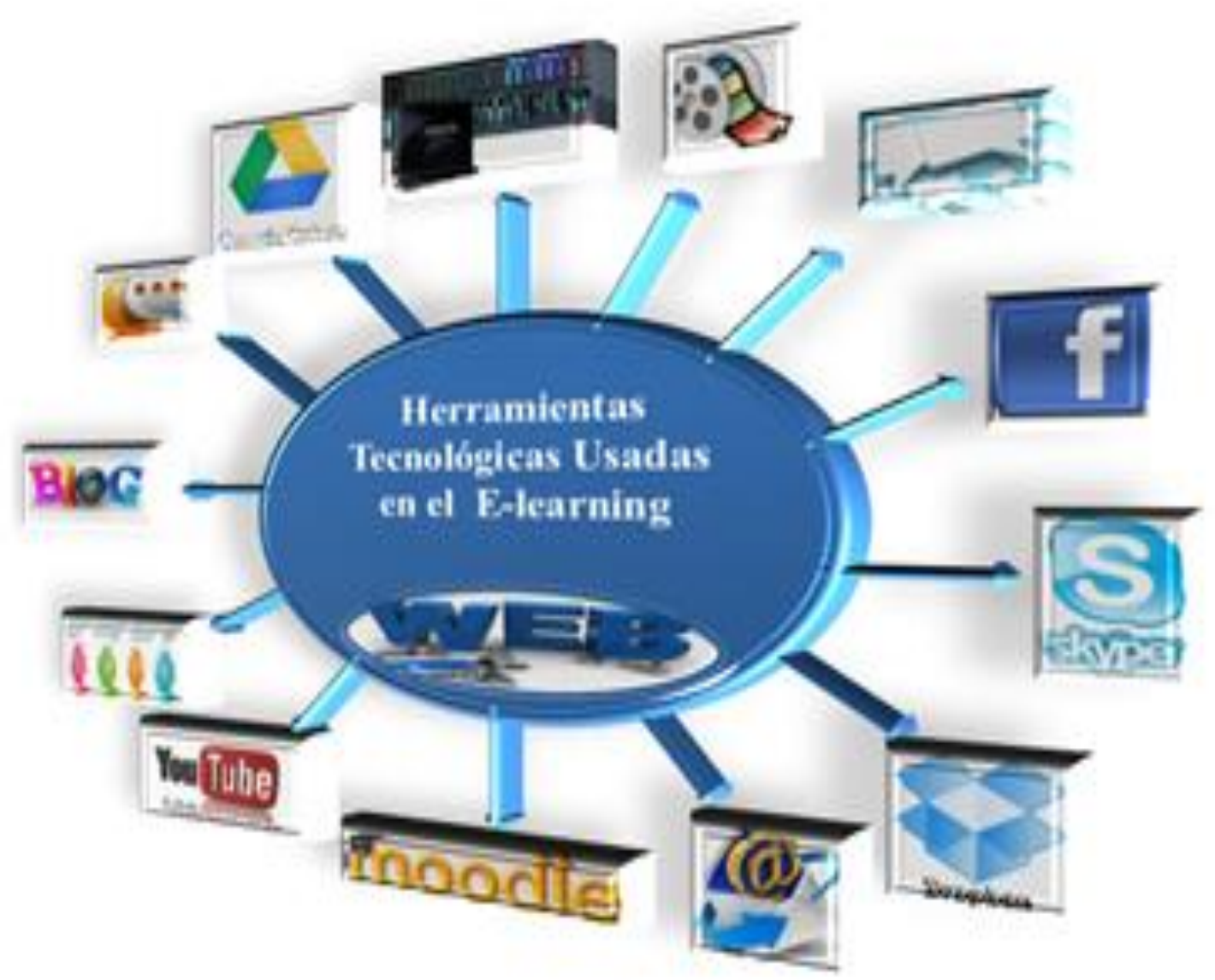

Figura 2. Herramientas tecnológicas usadas en el E-learning. (Fuente: Elaboración por el autor)

\section{Cursos a distancia ventajas y desventajas}

La educación a distancia no es un fenómeno reciente; en realidad ha sido un modo de enseñar y aprender de millares de personas durante más de un siglo. Es allí donde el educando y el educador se encuentran en un espacio físico o geográfico distante y no es impedimento para lograr la comunicación por medio de las TIC y lograr así llegar al aprendizaje.

Los estudiantes manifestaron que en ciertos momentos se ve truncado el proceso de instrucción por la mala calidad del internet que poseen en sus casas por su velocidad muy baja para navegar en la web y en su universidad no poseen la conexión para ellos poder trabajar mejor y cumplir con sus actividades pautadas. Explicando también de las interrupciones esporádicas y de caída de la conexión por lapsos amplios y por otros factores como las interrupciones eléctricas. Podría ser estos, uno de los factores que pudieran interferir en las ventajas y desventajas de los cursos que se administran bajo la modalidad a distancia que más adelante se explicarán detalladamente.

\section{CONCLUSIONES}

La mayoría de los profesores y los estudiantes emplean las herramientas de las TIC en los procesos educativos a distancia. Se destaca de esta manera la importancia del uso de las mismas en el contexto pedagógico.

Una cantidad importante de profesores son capaces de adaptar el uso de las herramientas de las TIC al plan de evaluación. Los estudiantes 
manifestaron que los cursos y el volumen de la información que se maneja bajo la modalidad a distancia, son adecuados.

Una forma de lograr la comunicación entre docente y estudiante en la instrucción a distancia es a través de las asesorías, como una alternativa, la cual es calificada como positivas. Sin embargo, sugieren la necesidad de que sea aumentada la frecuencia y actividad de estos encuentros virtuales. Se destaca la importancia del trabajo docente ya que este actúa como un guía al brindar ayuda a sus estudiantes a través de las asesorías. Por su parte, el trabajo del estudiante es como constructor de su propio aprendizaje.

Se deduce del estudio que una manera para que el estudiante participe de manera activa es con la modalidad a distancia. Estos aseguraron que les permite intervenir de manera constante, lo que les da la oportunidad de tener una visión crítica frente al tema trabajado y poder adecuar el proceso de enseñanza a su propio ritmo.

La gran mayoría de los estudiantes usan los medios electrónicos en los procesos de enseñanza y aprendizaje sin embargo manifestaron desconocer que este proceso se denomina E-learning.

La totalidad de los profesores concibió el $E$ learning como la forma de educar a través de Internet. Además de que tienen claro que con el E-learning se usan las herramientas electrónicas con fines educativos y que se puede dar de manera sincrónica, asincrónica o mixta; que a pesar de que no busca sustituir a la educación formal, saben que representa un medio a través del cual se rompen las barreras de espacio, tiempo y recursos, para así lograr el buen desarrollo de los procesos de enseñanza y aprendizaje.
Los cursos a distancia proporcionan a los docentes y a los estudiantes la posibilidad de interrelacionarse para desarrollar un aprendizaje colaborativo en vista de que intercambian información y se puede construir y reforzar el conocimiento. Sin embargo, para que esto sea así, es necesario que exista armonía entre el trabajo del docente como instructor, el contenido a enseñar y la actividad del estudiante. Es por ello que los cursos a distancia tienen ventajas y desventajas desde las percepciones de educadores y educandos.

Las principales ventajas, tanto para los docentes como para los estudiantes, están asociadas con la disminución de costos y uso de recursos, así como mejor administración del tiempo, mayor participación, es decir, le da al usuario la oportunidad de que realice su instrucción en un ambiente flexible y sin barreras, lo que favorece la comunicación. Tales condiciones, según el profesorado de la universidad, benefician al estudiante, quien mediante el uso de las herramientas tecnológicas logran su prosecución académica.

Para los docentes existen ciertas dificultades para impartir los cursos a distancia en la universidad, principalmente porque no se cuenta con un buen acceso a internet. Por esta razón, deben trabajar desde sus hogares $u$ otros sitios y con sus propios recursos, lo que implica mayor tiempo para observar y revisar las asignaciones hechas a los estudiantes.

La conexión a la red es una desventaja presente en el "Siso Martínez", por su lentitud lo que baja su capacidad de carga y por ende el desempeño eficiente de las actividades planificadas con las plataformas ya señaladas. 
Adicionalmente, los docentes manifestaron que también existen otras debilidades que no dependen de la conexión en la institución, tales como: a) problema de conexión en su hogar, pues tienen que usarlo por no contar con acceso de internet en la universidad y por ende el docente deben destinar mayor tiempo y observación para la revisión de materiales y trabajos; b) la poca interacción entre estudiantes y el profesorado; y c) la inexperiencia del educador y educando en el manejo de las herramientas tecnológicas.

\section{REFERENCIAS}

Albornoz, O. (1999). Las políticas educativas en el segundo quinquenio presidencial de Rafael Caldera. Caracas. Venezuela: Ediciones Faces/UCV

Arias, F. (2012). El proyecto de investigación. Introducción a la metodología cientíica. Caracas. Venezuela: Editorial Episteme, C.A

Bermejo, S. (2005). Cooperative electronic learning in virtual labs throungh forums. IEEE Transactions on Education, 48(1), 140-149

Castro, S., Casado, D. y Guzmán, B. (2007). Las Tic en los procesos de Enseñanza y Aprendizaje. Revista Laurus, 13(23). Recuperado de redalyc.uaemex.mx/pdf/761/76102311.pdf

Contreras, N. (2008). La enseñanza-aprendizaje de lenguas extranjeras y las TICS: El caso del español como Lengua Extranjera (ELE). Iniciación a la Investigación, Especial, I-VII Recuperado de http: //revistaselectronicas.ujaen.es/index.php/inin v/article/view/233/214

Cook, T. y Reichardt, Ch. (1997). Métodos cualitativos y cuantitativos en investigación evaluativa. Madrid: Ediciones Morata

Darías, V. (2001). La tecnología en la escuela Venezolana. Candidus, 3(16), 19-20

De Marco, P. (2015). La autorregulación del aprendizaje en la modalidad mixta en la Universidad Pedagógica Experimental
Libertador- Instituto Pedagógico de Caracas (UPEL-IPC) una propuesta teórica para su implementación (tesis doctoral). Instituto Pedagógico de Caracas, Universidad Pedagógica Experimental Libertador

Duart J. y Sangrà A. (2000) Aprendizaje y virtualidad: ¿un nuevo paradigma formativo? Recuperado de www.terras.edu.ar/.../BDUART-JosephSANGRA-Alber

Fernández, R., Server, P. y Carballo, C. (2006). Aprendizaje con nuevas tecnologías paradigma emergente. ¿Nuevas modalidades de aprendizaje? EDUTEC. Revista Electrónica de Tecnología Educativa, 20, 1-24. Recuperado de http://edutec.rediris. es/Revelec2/revelec20/raul20.pdf

García-Peñalvo, J. y García C, J. (2001). Los espacios virtuales educativos en el ámbito de Internet: un refuerzo a la formación tradicional. Recuperado de http://campus.usal.es/ teoriaeducacion/rev numero_06_2/n6_02_art_garcia_penalo.htm

Garrison, D. R. (1985). Three generations of technological innovation in distance education. Distance Education, 16, 235-241

González-Videgaray, M. (2007). Evaluación de la reacción de alumnos $y$ docentes en un modelo mixto de aprendizaje para Educación Superior. Recuperado de http://www.uv.es/RELIEVE/v13n1/RELIEVEv13 n1_4.htm

Guzmán, B. (2013) Evaluación del Curso Tecnología de Información y Comunicación y Educación Ambiental a Través de sus Productos (trabajo de ascenso para optar a la categoría de profesor Titular). Universidad Pedagógica Experimental Libertador, Instituto Pedagógico de Caracas, Venezuela

Guzmán B., (2008). Los docentes de la universidad pedagógica experimental libertador-instituto pedagógico de Caracas ante las tecnologías de información $y$ comunicación Investigación y postgrado, ISSN 1316-0087, 23(1), 37-72 
Guzmán, B., (2005). Actitudes de los docentes de la Universidad Pedagógica Experimental Libertador ante las Tecnologías de Información y Comunicación (tesis doctoral). Universidad Pedagógica Experimental Libertador, Instituto Pedagógico de Caracas, Venezuela

Hernández, M. (2010). Las tutorías, los tutores, los asesores. En: Educación a distancia: actores y experiencias. Tomo II. Loja: UTPL

Hollan, J., Hutchins, E., y Kirsch, D. (2000). Distributed Cognition: Toward a New Foundation for $\mathrm{HCl}$ Research. ACM Transactions on Computer Human Interaction, 17,174-196

Iniciarte, M. (2007). Gestión Docente en la Administración de Educación a Distancia. Recuperado de http://revistas.upel.ve/index.php/educare/arti cle/dowload/57/51

Kustcher, N. y St.Pierre A. (2001). Pedagogía e Internet Aprovechamiento de las Nuevas Tecnologías. México, D.F: Editorial Trillas

Marcelo, C. (2002). Conceptos en torno a la teleformación. E, C. Marcelo et al. (Coord.): Elearning-Teleform@ción.Diseño, desarrollo y evaluación de la formación a través de internet. Barcelona: Gestión 2000

Martínez M. (2007). La investigación cualitativa etnográfica. Manual teórico-práctico. México D.F.: Trillas.

Pérez, A. (2009). Guía metodológica para anteproyectos de investigación. Caracas: Fedeupel

Pizarro G., y Cordero D. (2013). Las TIC: Una herramienta tecnológica para el desarrollo de las competencias lingüísticas en estudiantes universitarios de una segunda lengua. Recuperado de:https://www.revistas.una.ac.cr/index.php/E DUCARE/article/download/5246/5458?inline $=1$ ? inline $=1$

Ruíz Bolívar, C. (1999). Las Nuevas Tecnologías de la Información y la Comunicación en la Concepción de una Pedagogía Alternativa para la Educación Superior. Conferencia presentada en el Congreso internacional de Pedagogía Alternativa Barquisimeto Venezuela

Sabino, C. (1999). El proyecto de investigación. Caracas: ORIAL

Tamayo y Tamayo, M. (1987). El proceso de investigación científica. México: Grupo Noriega Editores

Universidad Pedagógica Experimental Libertador [UPEL] (2016). Reglamento del Personal Académico de la UPEL. Resolución no. 2016.453.1032, diciembre 2016

Williams, P., Nicholas, D. y Gunter, B. (2005). " $E$ learning: what the literatur tells us about distance education - An overview". Aslib Proceedings, 572), 109-122

Yegres, A. (2000). Ética y formación docente. Instituto Universitario Pedagógico Monseñor Arias Blanco. Caracas: Autor

Zenger, J. T. y Walker, T. J. (2000). Impact of the internet on entomology teaching and research. Annual Review of Entomology (45), 747-767 